\title{
NIL SUBRINGS OF ENDOMORPHISM RINGS OF MODULES
}

JOE W. FISHER

\begin{abstract}
Let $M$ be an $R$-module and let $\operatorname{End}_{R}(M)$ be the ring of all $R$-endomorphisms of $M$. If $M$ is Artinian, then each nil subring of $\operatorname{End}_{R}(M)$ is nilpotent. If $M$ is Noetherian, then the indices of nilpotency of the nil subrings of $\operatorname{End}_{R}(M)$ are bounded.
\end{abstract}

Introduction. In [6] Procesi and Small showed that if $M$ is a Noetherian $R$-module and if $R$ satisfies a polynomial identity, then each nil subring of $\operatorname{End}_{R}(M)$ is nilpotent. Later Small [4] removed the hypothesis on $R$ and proved that if $M$ is Noetherian, then each nil subring of $\operatorname{End}_{R}(M)$ is nilpotent. This raises the dual question: If $M$ is Artinian, then is each nil subring of $\operatorname{End}_{R}(M)$ nilpotent? We give a proof which does provide an affirmative answer; moreover, it can be immediately dualized to give a proof of Small's theorem.

$\S 2$ is an appropriate place to include the following joint result of the author and Lance W. Small (who kindly gave his permission to me to publish it here): If $M$ is a Noetherian $R$-module, then the indices of nilpotency of the nil subrings of $\operatorname{End}_{R}(M)$ are bounded. In proving this we also show that if $M$ is an injective Noetherian $R$-module, then the Jacobson radical of $\operatorname{End}_{R}(M)$ is nilpotent. Putting this together with wellknown results, we obtain that the endomorphism ring of an injective Noetherian module is semiprimary.

At the end of the paper the author poses two questions which arise from this work.

1. Endomorphism rings of Artinian modules. Throughout this paper, $R$ will denote an associative ring which does not necessarily have a unity and $M$ will denote a right $R$-module.

Lemma 1.1. If $M$ is an Artinian $R$-module, then $S=\operatorname{End}_{R}(M)$ satisfies the ascending chain condition on left annihilators.

Proof. We note that $M$ becomes a faithful left $S$-module via $\phi \cdot m=$ $\phi(m), \phi \in S, m \in M$. In order to show that $S$ satisfies the ascending chain

Received by the editors October 26, 1971.

AMS 1970 subject classifications. Primary 16A22, 16A34; Secondary 16A64, 16A52.

Key words and phrases. Nil ring, T-nilpotent ring, nilpotent ring, endomorphism ring, Artinian module, Noetherian module, injective Noetherian module.

(c) American Mathematical Society 1972 
condition on left annihilators, we will show equivalently that $S$ satisfies the descending chain condition on right annihilators. Suppose that $A_{1} \supset A_{2} \supset \cdots \supset A_{i} \supset \cdots$ is a descending chain of right annihilators in $S$. Then $A_{1} M \supset A_{2} M \supset \cdots \supset A_{i} M \supset \cdots$ is a descending chain of $R$-submodules of $M$. Hence at some point $A_{k} M=A_{k+1} M=\cdots$. If $\phi A_{k+1}=0$ with $\phi \in S$, then $\phi A_{k} M=0$. Since $M$ is a faithful $S$-module, $\phi A_{k}=0$. Therefore $A_{k}=A_{k+1}$.

A ring $R$ is said to be left T-nilpotent if for each sequence $\left\{x_{n}\right\}$ of elements in $R$ there exists an $n$ such that $x_{1} x_{2} \cdots x_{n}=0$.

LEMMA 1.2. Let $S$ be a ring which satisfies the ascending chain condition on left (right) annihilators. Then a subring $N$ of $S$ is nilpotent if and only if $N$ is left (right) T-nilpotent.

Proof. See Fisher [1, Proposition 1.5].

The following lemma is contained in the proof of Fisher [1, Theorem $1.1]$.

LEMMA 1.3. Let $S$ be a ring which satisfies the ascending chain condition on left annihilators and let $N$ be a nil subring of $S$ which is not left $T$-nilpotent. Then there exists a sequence $\left\{x_{n}\right\}$ of elements in $N$ such that (a) $x_{1} x_{2} \cdots x_{n} \neq 0$ for each $n$, and (b) $x_{1} x_{2} \cdots x_{n} x_{i}=0$ for each $n \geqq i$.

Proof. We say that $x_{1} \in N$ has an infinite chain if there exists a sequence $\left\{x_{t}\right\}$ in $N$ such that $x_{1} x_{2} \cdots x_{t} \neq 0$ for each $t$. Since $N$ is not left $T$-nilpotent, there exist elements in $N$ which have an infinite chain. Let $l\left(x_{1}\right)$, the left annihilator of $x_{1}$, be maximal in $\{l(y): y \in N$ has an infinite chain $\}$. Inductively we find $x_{n}$ such that $l\left(x_{n}\right)$ is maximal in $\{l(y): y \in N$ and $x_{1} x_{2} \cdots x_{n-1} y$ has an infinite chain $\}$. It is now easy to verify that $l\left(x_{i}\right)=l\left(x_{i} x_{i+1} \cdots x_{i+j}\right)$ for each $i$ and $j$.

We claim that $x_{1} x_{2} \cdots x_{n} x_{i}=0$ for each $n \geqq i$. If $x_{1} x_{2} \cdots x_{n} x_{i} \neq 0$, then $x_{1} x_{2} \cdots x_{n} x_{i} x_{i+1} \cdots x_{k} \neq 0$ for each $k$ since $l\left(x_{i}\right)=l\left(x_{i} x_{i+1} \cdots x_{k}\right)$ for each $k$. Hence $x_{1} x_{2} \cdots x_{i-1} x_{i} x_{i+1} \cdots x_{n} x_{i}$ has an infinite chain. It follows from the maximality of $l\left(x_{i}\right)$ that $l\left(x_{i} x_{i+1} \cdots x_{n} x_{i}\right)=l\left(x_{i}\right)$. However this is impossible since $x_{i} x_{i+1} \cdots x_{n}$ is nilpotent.

LEMMA 1.4. Let $X, Y, Z$ be submodules of $M$. If $X \subset Y, X+Z=Y+Z$, and $X \cap Z=Y \cap Z$, then $X=Y$.

Proof. Evident.

Now we come to the main theorem in this paper which was announced in Fisher [3].

THEOREM 1.5. If $M$ is an Artinian R-module, then each nil subring of $\operatorname{End}_{R}(M)$ is nilpotent. 
Proof. Let $N$ be a nil subring of $S=\operatorname{End}_{R}(M)$. We claim that $N$ is left $T$-nilpotent, and therefore nilpotent by Lemma 1.1 and Lemma 1.2. Suppose that $N$ is not left $T$-nilpotent. Then it follows from Lemma 1.3 that there exists a sequence $\left\{\phi_{n}\right\}$ of elements in $N$ such that (a) $\phi_{1} \phi_{2} \cdots \phi_{n} \neq 0$ for each $n$, and (b) $\phi_{1} \phi_{2} \cdots \phi_{n} \phi_{i}=0$ for each $n \geqq i$. Set $s_{j}=\phi_{1} \phi_{2} \cdots \phi_{j}$ for each $j$. Then $s_{1} M \supset s_{2} M \supset \cdots \supset s_{j} M \supset \cdots$ is a descending chain of $R$-submodules of $M$. Hence at some point $s_{k} M=$ $s_{k+1} M=\cdots$. Whence $M=\operatorname{Ker} s_{i}+\phi_{i+1} M$ for each $i \geqq k$ since for each $m \in M$ there exists $m^{\prime} \in M$ such that $s_{i} m=s_{i+1} m^{\prime}$ and $m=\left(m-\phi_{i+1} m^{\prime}\right)+$ $\phi_{i+1} m^{\prime}$. From (b) we have that for each $i \geqq k, \phi_{i+1} M \subset \operatorname{Ker} s_{n}$ for each $n>i$. Thence $M=\operatorname{Ker} s_{i}+\bigcap_{n>i} \operatorname{Ker} s_{n}$ for each $i \geqq k$. It follows from this and Lemma 1.4 that the following chain is a strictly descending chain of $R$-submodules of $M$ :

$$
\operatorname{Ker} s_{k} \supset\left[\operatorname{Ker} s_{k} \cap \operatorname{Ker} s_{k+1}\right] \supset \cdots \supset\left[\bigcap_{i=k}^{t} \operatorname{Ker} s_{i}\right] \supset \cdots .
$$

This contradiction proves the claim and therefore the theorem.

2. Endomorphism rings of Noetherian modules. The results in this section were obtained jointly with Lance Small. The following theorem is due to Lance Small and appears in Goldie-Small [4].

THEOREM 2.1. If $M$ is a Noetherian $R$-module, then each nil subring of $\operatorname{End}_{R}(M)$ is nilpotent.

Proof. Dualize the proof of Theorem 1.5 or see Goldie-Small [4].

We will proceed to show that if $M$ is a Noetherian $R$-module, then the indices of nilpotency of the nil subrings of $\operatorname{End}_{R}(M)$ are bounded. For the remainder of this section we let $S=\operatorname{End}_{R}(M)$ and define $Z=\{\phi \in S: \operatorname{Ker} \phi$ is an essential $R$-submodule of $M\}$. An $R$-module $M$ is said to have finite dimension $n$ if $M$ contains a direct sum of $n$ nonzero $R$-submodules and each direct sum of nonzero $R$-submodules of $M$ has at most $n$ terms.

THEOREM 2.2. If an $R$-module $M$ has dimension $n$, then each ascending chain and each descending chain of left annihilators in $S / Z$ have less than $n+1$ strict inclusions.

Proof. See the proof of Fisher [2, Theorem 1.3].

COROLlaRY 2.3. If an $R$-module $M$ has dimension $n$, then each nil subring of $S / Z$ is nilpotent and the indices of nilpotency of the nil subrings of $S / Z$ are bounded by $n+1$. Moreover, if $Z$ is nilpotent with index of nilpotency $k$, then each nil subring of $S$ is nilpotent and the indices of nilpotency of nil subrings of $S$ are bounded by $k(n+1)$.

Proof. See the proof of Fisher [2, Corollary 1.5]. 
Proposition 2.4. If $M$ is a Noetherian $R$-module, then $Z$ is nilpotent.

Proof. First we note that $S$ satisfies the ascending chain condition on right annihilators by Procesi-Small [6, Lemma]. Thus by Lemma 1.2 it suffices to show that $Z$ is right $T$-nilpotent.

Let $\left\{\phi_{n}\right\}$ be a sequence of elements in $Z$. Set $s_{j}=\phi_{j} \cdots \phi_{2} \phi_{1}$. Then Ker $s_{1} \subset \operatorname{Ker} s_{2} \subset \cdots \subset \operatorname{Ker} s_{j} \subset \cdots$ is an ascending chain of $R$-submodules of $M$. Hence at some point $\operatorname{Ker} s_{k}=\operatorname{Ker} s_{k+1}=\cdots$. From this we obtain that $s_{k} M \cap \operatorname{Ker} \phi_{k+1}=\{0\}$. Since $\operatorname{Ker} \phi_{k+1}$ is an essential $R$-submodule of $M$, it follows that $s_{k} M=0$. Wherefore $s_{k}=\phi_{k} \cdot \phi_{2} \phi_{1}=0$. Therefore $Z$ is right $T$-nilpotent.

COROllary 2.5. If $M$ is an injective (quasi-injective) Noetherian $R$ module, then $\operatorname{End}_{R}(M)$ is semiprimary.

Proof. It is well known [5] that $\operatorname{End}_{R}(M)$ is semiperfect and that $Z$ is the Jacobson radical of $\operatorname{End}_{R}(M)$. Since $Z$ is nilpotent, it follows that $\operatorname{End}_{R}(M)$ is semiprimary.

THEOREM 2.6. If $M$ is a Noetherian $R$-module, then the indices of nilpotency of the nil subrings of $\operatorname{End}_{R}(M)$ are bounded.

Proof. The result follows from Proposition 2.4 and Corollary 2.3.

We conclude this paper by posing the following two questions: (1) Do there exist injective Noetherian $R$-modules which are not Artinian? and (2) If $M$ is an Artinian $R$-module, then are the indices of nilpotency of the nil subrings of $\operatorname{End}_{R}(M)$ bounded as they are in the Noetherian case? If, in addition, $M$ is finitely generated, then the answer to (2) is affirmative.

\section{REFERENCES}

1. J. W. Fisher, On the nilpotency of nil subrings, Canad. J. Math. 22 (1970), 12111216. MR 42 \#3115.

2. - Nil subrings with bounded indices of nilpotency, J. Algebra 19 (1971), $509-516$.

3. - Endomorphism rings of modules, Notices Amer. Math. Soc. 18 (1971), 619-620. Abstract \#71T-A85.

4. A. W. Goldie and L. W. Small, A note on rings of endomorphisms (to appear).

5. J. Lambek, Lectures on rings and modules, Blaisdell, Waltham, Mass., 1966. MR 34 \#5857.

6. Claudio Procesi and Lance Small, Endomorphism rings of modules over PI-algebras, Math. Z. 106 (1968), 178-180. MR 38 \#2167.

Department of Mathematics, University of TeXas, Austin, TeXas 78712 